\title{
Umbrales para la determinación de la brecha digital: comparativa entre regiones desarrolladas
}

\author{
Threshold for the determination of the digital breach: \\ Comparative among developed regions
}

Juan-José MENDOZA-RUANO'1

Jorge CALDERA-SERRANO²

\section{Resumen}

Este trabajo tiene como objetivo principal establecer una escala porcentual que sirva de base metodológica para determinar la existencia o no de brecha digital entre regiones tecnológicamente desarrolladas. Una vez analizada la literatura científica observamos un claro déficit al no contar con una base estudiada que determine bajo qué parámetros se puede hablar de Brecha Digital al realizar la comparativas entre zonas. Además, contextualizamos el tema con una breve descripción de los conceptos básicos para el estudio, como son el de Sociedad de la Información, y en mucha mayor profundidad, la Brecha Digital. Las escalas planteadas son válidas para zonas geográficas de un nivel desarrollado en tecnologías de la información y la comunicación, no siendo un trabajo válido para zonas subdesarrolladas o en vías de transformación tecnológica. Se plantea por tanto cuatro niveles de brecha digital que corresponden con las siguientes escalas porcentuales: 1\%-5\%, 6\%-10\%, 11\%-15\%, más de $16 \%$, respectivamente.

Palabras Ilave: Brecha digital. Desigualdad social. Herramienta metodológica. Sociedad de la Información.

\begin{abstract}
This piece of work has a main goal: setting a percentage scale that serves as a methodological basis in order to determine the digital breach existence or not among regions technologically developed. Once the scientific literature is analysed we can observe a clear deficiency because we don not have a studied basis that can determine under which parameters we can talk about Digital Breach when we are comparing different regions. Moreover, we contextualize the issue with a brief description of the basic concepts for the study, such as the Information Society issue, and in a deeper way, the Digital Breach. The scales suggested are valid and acceptable for geographical zones with a high level on Information and Communication Technologies, not being an acceptable approach for underdeveloped regions or starter regions with the technological transformation. Hence, four levels of digital breach are suggested that are suitable with the percentage scales: $1 \%-5 \%, 6 \%-10 \%, 11 \%-15 \%$, more than 16\%, respectively.
\end{abstract}

Keywords: Digital divide. Social inequality. Methodolgical tools. Information society.

\section{Introducción}

En las últimas décadas, las nuevas Tecnologías de la Información y la Comunicación (TIC) han impactado en nuestra sociedad, logrando modificar nuestra manera de vivir, de comunicar, de producir y de comercializar. Estas nuevas tecnologías nacen gracias a la convergencia de la electrónica, el software y las infraestructuras de las

\footnotetext{
1 Universidad de Extremadura, Facultad de Ciencias de la Documentación y la Comunicación. Badajoz, España.

2 Universidad de Extremadura, Facultad de Ciencias de la Documentación y la Comunicación, Departamento de Información y Comunicación. Plazuela de Ibn Marwan, s/n., 06001, Badajoz, España. Correspondencia a nombre de/Correspondence to: J.-J. CALDERA-SERRANO. E-mail:<jcalser@alcazaba.unex.es>. Recibido el dia 4/6/2013, re-apresentado em 27/11/2013 y aceptado para su publicación el 26/2/2014.
} 
telecomunicaciones, lo que conocemos como revolución digital; esto es, el conjunto de innovaciones tecnológicas que han hecho posible que la voz y el sonido, la imagen y los datos puedan transmitirse de un lugar a otro a gran velocidad reduciendo toda su carga de información a simples combinaciones de "ceros" y "unos" integrados dentro de un circuito electrónico.

La asociación de estas tecnologías da lugar a una nueva concepción del proceso de la información, en el que las comunicaciones abren nuevos horizontes y paradigmas; la revolución digital se convierte así en el elemento crítico que ha permitido todas estas transformaciones que vive el mundo contemporáneo.

La información cobra mayor relevancia y se convierte en un factor determinante en todos los procesos, desempeñando un papel vertebrador en la denominada Sociedad de la Información.

La Sociedad de la Información es una forma de desarrollo económico y social en que la adquisición, almacenamiento, procesamiento, evaluación, transmisión, distribución y diseminación de la información con vistas a la creación de conocimientos y a la satisfacción de las necesidades de las personas y de las organizaciones, es utilizada intensivamente en tanto que elemento determinante en la vida económica, social, cultural y política (Unión Europea, 1997).

Sin lugar a dudas, las nuevas Tecnologías de la Información y la Comunicación han desempeñado un papel medular en el desarrollo de la Sociedad de la Información y son instrumentos que pueden contribuir a alcanzar los objetivos trazados por las personas y las organizaciones, aumentando así la posibilidad de interacción y comunicación, y la mejora de la calidad de vida económica, social y cultural.

La irrupción de las TIC ha transformado la imagen del mundo, en especial la implantación de Internet en todas y cada una de las facetas de nuestra vida. Internet se ha convertido en el elemento más significativo de la denominada Sociedad de la Información y del Conocimiento, ya que permite en primera instancia, comunicar instantáneamente a todo el mundo (conectado). Es tal la repercusión que actualmente tiene Internet en muchas de nuestras decisiones y actuaciones diarias, que se ha convertido en el punto esencial desde el que analizar y valorar al fenómeno de la Brecha Digital.

No obstante, no todo son bondades en esta revolución informática. De ahí, que estas nuevas tecnologías estén planteando nuevos desafíos y retos a la sociedad, especialmente los referidos a cómo aprovechar los beneficios de estas tecnologías, cómo hacer que todos, sin discriminación alguna, participemos de estos beneficios, sobre todo aquellos sectores de población, aquellas áreas geográficas, y aquellas zonas del planeta más necesitadas de los beneficios de esta revolución tecnológica.

Día a día vemos cómo aparecen nuevas formas de exclusión propiciadas precisamente por estas tecnologías, ya que no todos consiguen hacer suyos los beneficios derivados de su aplicación. De hecho, ya es posible afirmar que la gran cantidad de información que permiten transferir, gestionar y facilitar está provocando una gran división entre los usuarios y los no usuarios de las mismas, entre los que saben y no saben utilizarlas, entre los que pueden y no pueden hacer uso de ellas, y todo ello a pesar de que los grandes precursores y defensores de estas tecnologías vaticinaban en sus orígenes que las diferencias entre las sociedades iban a desaparecer con la aplicación de las mismas ya que, se supone, nos iban a hacer a todos la vida mucho más fácil. Sin embargo, existen sectores de población y zonas no ya solo del mundo, sino dentro de un mismo país, desprovistos de estas nuevas oportunidades que han traído las nuevas tecnologías.

Este fenómeno, denominado Brecha Digital, no es otra cosa que las desigualdades que existen entre las personas, comunidades o países que tienen acceso y uso de las TIC y, sobre todo, de Internet como una parte rutinaria de su vida diaria y aquéllas que no tienen acceso a las mismas y que, aunque lo tengan, no saben utilizarlas de manera óptima para su beneficio.

La Brecha Digital es una nueva dimensión de la amplia y vieja brecha social, que perpetúan las relaciones de poder, asociada con factores económicos, geográficos, raciales, culturales, educativos, de género y de edad entre otros. El problema que la Brecha Digital representa para la humanidad debe ser motivo de profunda preocupación para todos los países del mundo. El ejercicio de la democracia depende fundamentalmente 
del acceso libre e ilimitado a la información. Es, por ello, preciso identificar estrategias que disminuyan el problema de la desigualdad en el acceso a la información.

\section{¿Qué es la brecha digital?}

La brecha digital es probablemente uno de los primeros conceptos con que se inicia la reflexión alrededor del impacto social de las Tecnologías de la Información y Comunicación. Desde entonces se percibe que estas tecnologías van a producir diferencias en las oportunidades de desarrollo de las poblaciones y que se establecerá una distancia entre aquellas que tienen o no tienen acceso a las mismas.

Existe una gran diversidad de enfoques, concepciones y definiciones de Brecha Digital. A continuación se presentan varias de ellas.

El World Resources Institute (2000) apoyado por varias de las más importantes empresas internacionales informáticas explica así esa hendidura que lejos de atenuar, está profundizando las desigualdades en el planeta. Se señala la existencia en todos los países de porcentaje de personas que tienen acceso a la mejor tecnología y a las mejores redes de información y comunicación, mientras que existe otro grupo que no tienen acceso a estas tecnologías, por aspectos derivados de múltiples factores (sociales, económicos, geográficos, laborales, etc.), y la diferencia entre estos dos grupos será la Brecha Digital.

La Organización para la Cooperación y Desarrollo Económico (2001) define la Brecha Digital como la distancia existente entre diferentes parámetros, tales como individuos, áreas geográficas, niveles socioeconómicos, etc., en relación a la oportunidad que éstos tienen para el acceso a las TIC y al uso de Internet, por lo que así se podrá determinar y apreciar diferencias tanto entre diversos países como en el marco de un único territorio.

Serrano Santoyo y Martínez Martínez (2003) la definen como la separación que existe entre las personas (comunidades, estados, países...) que utilizan las TIC como una parte rutinaria de su vida diaria y aquellas que no tienen acceso a las mismas y aunque las tengan no saben cómo utilizarlas.
DiMaggio et al. (2001) asumen que las desigualdades en el uso de Internet se producen entre los que tienen y los que no tienen acceso a Internet, y otros como Bucy (2000) y Van Dijk (2002) que definen las desigualdades no solamente como resultado del acceso potencial a Internet sino también como las diferencias en el uso de esta tecnología.

Monge Gonzaléz y Chacón (2002) del Departamento de Comercio de los United States (EE.UU), señalan que existe un grupo de personas que cuentan con equipos informáticos, con acceso a un servicio telefónico de calidad lo que se traduce en rapidez de acceso a la red, y que además existe una riqueza de contenidos en la información accesible por medio de la red. Además este grupo de persona cuenta con una capacitación tecnológica (alfabetización digital) que hace posible el obtener buenos rendimientos del acceso a la red. Los autores lo contraponen con otro grupo que ni tienen las mejores computadoras, ni tienen acceso a la red, ni aquellos que acceden pueden obtener los mejores contenidos. La diferencia entre los dos grupos sería la Brecha Digital.

Más recientemente, el estudioso costarricense Monge González y Hewit (2004) consideran que el concepto de Brecha Digital está relacionado con el acceso que las personas tienen a las TIC, tales como el acceso mismo, las herramientas tecnológicas, la calidad del uso que se realiza sobre estas tecnologías y el impacto que el uso de ésta proporciona en el bienestar del individuo.

En estas definiciones un tanto genéricas del concepto se recogen algunas de las diferentes dimensiones de la Brecha Digital.

Otros estudiosos del tema han delimitado dichas dimensiones a través de conceptualizaciones separadas. Así, por ejemplo Norris (2001) evidencia la multidimensionalidad del concepto mediante la consideración de tres tipos de brechas: la global, la democrática y la social. La primera de ellas se centra en una brecha de acceso a la tecnología, y muy especialmente a Internet. Esta diferencia vendrá marcada por un mundo a varias velocidades de uso e implantación de las tecnologías, por lo que la diferencia será clara entre países industrializados y aquellos que están en vías de desarrollo. En segundo lugar, Norris da cuenta de la 
dimensión política y participativa de la brecha, definiendo la brecha democrática como la diferencia en el uso de las tecnologías entre aquellos la optimizan para participar en la vida pública y aquellos que no pueden o no saben. Por último, la brecha social es considerada como aquella que se da dentro de un mismo país o sociedad, como resultado de su estratificación social o geográfica interna. Este tipo de Brecha Digital se hace eco de las diferencias en el acceso entre distintas regiones, grupos sociales y entre individuos según su capacidad económica, su sexo, nivel educativo, raza o lugar de residencia (brecha geográfica).

Van Dijk y Hacker (2003) entienden también que la Brecha Digital es una expresión más de las diferencias sociales existentes en un determinado territorio y que, por lo tanto, dependen de las características sociodemográficas de dicho territorio. Mientras que estos y otros autores mantienen una concepción mucho más pesimista según la cual las diferencias en el acceso y uso de las TIC disminuirán en la misma medida en que lo hagan las diferencias sociales y estructurales existentes. Otros, como Anderson et al. (2001) o Compaine (2001) vuelven al carácter original y distintivo de la Brecha Digital que surge como consecuencia de las relaciones entre ciudadanos y tecnología, para afirmar que la extensión de Internet hará posible la desaparición de la Brecha Digital y permitirá que triunfe la descentralización, los procesos de globalización, la armonización y empoderamiento rápido.

Otros autores definen la Brecha Digital como analfabetismo digital, el cual consiste en la escasa habilidad o competencia de algunas personas para manejar las computadoras, Internet y las tecnologías en general. Es decir, la Brecha Digital está relacionada con aspectos de acceso a los beneficios de la digitalización y también a las capacidades de una población o grupo social para utilizar las TIC de manera apropiada y que contribuya al desarrollo sostenible.

Aunque todas las definiciones contienen unas ideas bastante similares, puede decirse que no existe hoy un único concepto sobre qué se entiende por Brecha Digital, sin embargo parece razonable afirmar que la existencia de una Brecha Digital está directamente relacionada con cuatro elementos, que son necesarios mencionar con el objeto de hacer un correcto diagnóstico del problema para poder luego plantear las medidas adecuadas para reducir y eliminar esa brecha. Estos son:

- La disponibilidad de un ordenador, u otro elemento de hardware que permita al ciudadano conectarse a Internet.

- La posibilidad de conectarse y poder acceder a la red, desde el hogar, el trabajo o la oficina.

- El conocimiento de las herramientas básicas para poder acceder y"navegar" en la red.

- La capacidad adecuada para hacer que la información accesible en la red pueda ser convertida en "conocimiento" por el usuario.

También apreciamos en las definiciones del concepto de Brecha Digital que se han ido modificando a través del tiempo. En un principio se refería básicamente a los problemas de conectividad. Posteriormente, se empieza a introducir la preocupación por el desarrollo de las capacidades y habilidades requeridas para utilizar las TIC (capacitación y educación) y últimamente también se hace referencia al uso de los recursos integrados en la tecnología. Así, el concepto de Brecha Digital incorpora los siguientes enfoques básicamente:

a) El enfoque hacia la infraestructura: o sea, la posibilidad/dificultad de disponer de ordenadores conectados a la red.

b) El enfoque hacia la capacitación: es decir, las habilidades y capacidades/dificultades de usar estas tecnologías. En este sentido, se comienza a desarrollar el concepto de alfabetización digital relacionado con el de Brecha Digital.

c) El enfoque hacia el uso de los recursos: se refiere a la limitación/posibilidad que tienen las personas para utilizar los recursos disponibles en la red. En los últimos tiempos, se ha integrado en el concepto de Brecha Digital las posibilidades de utilizar la tecnología no solamente para acceder a la información, al conocimiento, sino también a un nuevo modo de educación y para aprovechar las "nuevas oportunidades" como el desarrollo de los negocios, la atención médica en línea, el teletrabajo, el disfrute de nuevas formas de entretenimiento y ocio.

Basados en estos elementos, muchos de los organismos internacionales han definido una política de 
desarrollo orientada a la reducción de la Brecha Digital. Sin embargo, a pesar de la evolución en el concepto, se enfatiza principalmente el desarrollo de la infraestructura tecnológica. Las inversiones y las políticas nacionales para la reducción de la Brecha Digital siguen orientadas principalmente hacia el desarrollo de la conectividad.

Para concluir, la Brecha Digital implica que hay una distribución no homogénea en el acceso a la infraestructura de comunicaciones, la conectividad y los contenidos digitales. Esta brecha se sustenta en desigualdades sociales preexistentes y además puede profundizar esas desigualdades. El nivel educativo, el estatus socio-económico, la ubicación geográfica, el género, la edad, la pertenencia a determinados grupos étnicos, la presencia de discapacidades, son todos aspectos que explican la exclusión digital de distintos colectivos y la agudización de su situación de exclusión social.

Pero la brecha más importante no se produce solamente en términos de acceso a la tecnología en sí misma; ya que las comunidades afectadas por la Brecha Digital de lo que quedan excluidas es de lo que estas tecnologías permiten hacer: la capacidad de procesar, seleccionar y producir información como un factor de desarrollo social. Por otra parte, los excluidos digitales no tienen la posibilidad de desarrollar un papel activo en la Sociedad de la Información y por tanto, no serán tomados en cuenta en su construcción. Esto implica que los valores y criterios del mercado que priman en el desarrollo de Internet y las telecomunicaciones los van a dejar cada vez más afuera. Con lo cual, la exclusión digital se agudiza, y con ella, una vez más, la exclusión social.

\section{Métodos}

En todo proceso de modernización, el cambio genera una nueva estructura de oportunidades, pero, conjuntamente, también cabe la posibilidad de que aparezcan nuevos tipos de desigualdad social o se acentúen las ya existentes. La transición a la Sociedad de la Información no es una excepción.

El objetivo del trabajo será definir los umbrales para la determinación de la Brecha Digital en unas zonas geográficas muy concretas y con características que sean similares, para que dichos umbrales puedan ser extrapolables y útiles para el análisis del fenómeno.

Cualquier investigación parte de un conocimiento que es preciso analizar. La ingente cantidad de literatura científica que versa sobre la Sociedad de la Información y la Brecha Digital, ha hecho que la revisión bibliográfica haya sido una labor importante. Dicha revisión bibliográfica nos ha llevado a identificar la inexistencia de parámetros porcentuales para determinar la Brecha Digital en la literatura científica.

El análisis de la realidad de la Comunidad Autónoma de Extremadura respecto a la media española ha sido realizada a partir de los datos del Instituto Nacional de Estadística (España, 2010) en su trabajo titulado "Encuesta sobre equipamiento y uso de tecnologías de la información y comunicación en los hogares"y en la "Encuesta sobre el uso de TIC y comercio electrónico en las empresas". Se han seleccionado 67 indicadores, analizados en un periodo de cuatro años (2006/2010).

Los indicadores empleados se han agrupado por bloques, que simplemente identificamos: Conexión a internet en hogares y motivos por los cuales no cuentan con dicha conexión; uso del ordenador y de éste con conexión a Internet (para qué y cuánto -asiduidad-); utilización y comunicación por medio del eadministración para hogares y ciudadanos. Para empresas se ha analizado la conexión a Internet; Quién utiliza la información; Para qué es utilizada la red.

La encuesta tenía como objetivo principal conocer el equipamiento tecnológico en los hogares y empresas, así como analizar su uso. Esta encuesta se realiza desde el 2004 de forma continuada en forma de "panel rotante" donde las mismas familias son analizadas durante varios años, aunque una cuarta parte de la población va cambiando en la muestra.

La muestra alcanza las 28.224 viviendas en 2004, siendo un número similar a lo largo de los años siguientes. En el año 2010 las familias analizadas fueron de 24.877 . La entrevista se realiza por teléfono. En caso de que dicha familia no cuente con teléfono la encuesta será presencial en la casa del analizado. La población objeto de estudio son principales familias, siendo exhaustivamente analizadas las personas con 16 años o más. 
La muestra es de modelo trietápico con estratificación de las unidades por medio de secciones censales. Por lo tanto, este muestreo es una representación de estratos por municipios y habitantes, siendo la selección de arranque de la muestra aleatorio.

El nivel de confianza del muestreo es del 95\% medida en términos de probabilidad.

Además de esta herramienta básica se han analizado otros dos fuentes. Estos han sido por un lado el documento de la Fundación Telefónica (2001) que realiza un informe sobre la Sociedad de la Información en España y los datos del Observatorio de la Sociedad de la Información del Gobierno de Extremadura (2009), con datos no actualizados desde 2009, pero que nos ha servido para contrastar y/o depurar algunos datos extraídos de la herramienta del INE.

\section{Determinación de umbrales}

Como ya hemos comentado anteriormente, la inexistencia de umbrales estadísticos para medir la Brecha Digital nos ha llevado a determinar dichos umbrales con el objeto de dotar al estudio de rigor científico.

Para ello, y siendo consciente que estos umbrales porcentuales son únicamente válidos para estructuras económicos-sociológicas-culturales-geográficas similares, los umbrales se han ajustado mucho partiendo de la premisa de la igualdad máxima entre iguales, teniendo presente que zonas geográficas con parecida renta per cápita, con niveles culturales semejantes, con cultura y sociedad parecidas, debieran contar con un parecido en acceso y uso a internet muy semejantes.

Por ello, los umbrales se han categorizado en cuatro niveles muy ajustados.

\section{- Brecha Digital Tipo 1}

Diferencial entre el 1\% al 5\% de la media. Puede estimarse que la Brecha Digital Tipo I es prácticamente un empate técnico en la mayor parte de los casos, ya que siempre habrá variaciones en cualquiera de las variables que hagan que exista una brecha digital o un desarrollo tecnológico mínimo respecto a la media (denominamos desarrollo tecnológico cuando cualquiera de los datos analizados están por encima de la media aritmética sobre las que estemos analizando).
Con ello no estamos señalando que no se deban realizar esfuerzos para eliminar dicha brecha y llegar a un desarrollo digital, lo que sí queremos señalar es que el diferencial porcentual es poco significativo además de poder ser fácilmente superable por medio de políticas de información y comunicación.

\section{- Brecha Digital Tipo /l}

Diferencial entre el 6\% al 10\% de la media. La Brecha Tipo II es una brecha que podríamos denominar de importancia media o moderada, y sobre la cual se debe actuar de forma más tajante. Un diferencial superior al 6\% ya es significativo en los contextos parejos con nivel de acceso y uso elevados. Las políticas estatales deben ir encaminadas a reducir dicho diferencial.

\section{- Brecha Digital Tipo III}

Diferencial entre el 11\% al 15\% de la media. Este tipo de Brecha es significativa y preocupante. Este diferencial con la media puede entenderse como una Brecha Digital real, con un diferencial que se traduce en problemas económicos, sociales; que se traduce en problemas para la creación de empresas y muy especialmente aquellas que cuentan con una base tecnológica. Además se traduce en diferencias significativas en las relaciones sociales de los individuos de la comunidad.

\section{- Brecha Digital Tipo IV}

Diferencial más del 16\% de la media. La Brecha Digital Tipo IV es sin lugar a dudas, preocupante. Una diferencia por encima del $16 \%$ se va a traducir en diferencias muy significativas en el entorno social, económico, etc., lo que provocará una fractura social con el resto de la sociedad española. Esta diferencia entre zonas de países del primer mundo y con un desarrollo tecnológico amplio debieran ser resueltas con políticas activas de eliminación de la brecha, ya sea desde el punto de vista de la implantación de las tecnologías de la comunicación e implantación social de tecnologías informáticas, o desde la perspectiva del uso de dichas tecnologías.

¿Bajo qué condiciones tendrían validez esta escala porcentual para medir la brecha digital?

Es muy importante determinar bajo qué condiciones es válido este umbral porcentual, siendo por tanto un estándar válido para investigaciones que puedan ser parecidas. 
Para ello hemos de determinar que ha sido testado para determinar la Brecha Digital en un periodo de 6 años de la Comunidad Autónoma de Extremadura, que está situada en un país como España que es aún considerado un país del primer mundo y con unas variables que la posicionan entre los países punteros desde el punto de vista tecnológico. Aunque actualmente España está modificando claramente muchas de las variables que la están empobreciendo, los datos se han analizado en el momento en que España estaba situada entre los ocho países del mundo con mayor pujanza económica. Además, la Comunidad Autónoma de Extremadura es la única comunidad española que sigue siendo objetivo uno en las ayudas de la Unión Europea para el desarrollo, ya que cuenta con altas tasas de paro y una renta por ciudadano bastante baja. Para contrarrestar estas variables negativas desde el Gobierno de Extremadura se han realizado en esos años grandes esfuerzos de acceso y capacitación tecnológica en toda la sociedad civil, además de incrementar el e-goverment de manera significativa.

Por lo tanto, los datos son equiparables para comparaciones entre zonas con alto nivel de acceso a las tecnologías de la información, con amplio desarrollo gubernamental para penetrar e introducir las tecnologías de la información entre sus ciudadanos y su administración como fórmula de comunicación, y que además están en contextos económicos y sociales desarrollados. No es significativo utilizar estos umbrales porcentuales para realizar un análisis entre estados de Norteamérica o de la Unión Europea con zonas o países de África, por ejemplo.

\section{Conclusión}

Siendo consciente de las limitaciones y complicaciones que se derivan de plasmar unos umbrales porcentuales para comparar realidades siempre dispares, se ha estimado conveniente contar con una herramienta de base, con un elemento metodológico estándar y claro para poder identificar claramente el problema derivado del desarrollo de la Sociedad de la Información como es la Brecha Digital.

Se estima importante contar con esta herramienta para dotar de valor metodológico a potenciales estudios posteriores, ya que de lo contrario la identificación de existencia de Brecha Digital vendrá marcada por intuiciones, que aunque bien intencionadas, no dejarán de ser estimaciones y sensaciones personales llevando a cabo la labor comparativa de dos o más realidades distintas. Esta labor intuitiva, aunque pueda contar con conocimientos previos y referencias que sirvan para la determinación de la existencia o no de la Brecha Digital, siempre estarán fundamentadas en datos porcentuales, por lo que es preferible que dicha labor vaya marcada desde la hipótesis teniendo en cuenta los datos porcentuales, y que sean las referencias culturales, sociológicas, económicas, geográficas, etc. las que después utilice el investigador para acabar de definir la existencia o no de la brecha.

Los cambios y revoluciones en la economía mundial hace también que sea necesario ajustar los elementos de comparación para analizarlos desde los iguales para que las comparaciones sean más justas y reales respecto de la realidad en la que se interconectan y comunican.

Y a partir de ahí, una vez determinada la Brecha Digital y conocido el entorno, será el momento de comenzar a buscar soluciones reales y que puedan ajustarse a la realidad, lo cual ha estado alejado muchas veces, al hacer comparaciones entre elementos con variables tan distintas que hacían esta comparación imposible o, al menos, poco útil.

\section{Agradecimientos}

Este trabajo ha sido financiado por la Junta de Extremadura (Consejería de Educación, Ciencia y Tecnología) y el Fondo Social Europeo dentro del plan de apoyo a las actuaciones de los Grupos de Investigación inscritos en el catálogo de la Junta de Extremadura. GR10019.

\section{Referencias}

Anderson, R.H. et al. Universal access to e-mail: Feasibility and cocietat implications. In: Compaine, B.M. (Ed.). The digital divide:

Facing a crisis or creating a myth? Cambridge: MIT Press, 2001, p.243-262. 
Bucy, E.P. Social access to the Internet. Harvard International Journal of Press/Politics, v.5, n.1, p.50-61, 2000.

Compaine, B.M. Declare the war won. In: Compaine, B.M. (Ed.). The digital divide: Facing a crisis or creating a myth? Cambridge: MIT Press, 2001. p.315-335.

DiMaggio, P. et al. Social implications of the internet. Annual Review of Sociology, v.27, p.307-336, 2001.

España. Instituto Nacional de Estadística. Encuesta sobre equipamiento y uso de tecnologías de la información y comunicación en los hogares. 2013. Disponible en: <http:// www.ine.es $/ \mathrm{jaxi} / \mathrm{menu}$. do? type $=$ pcaxis $\&$ path $=\% 2 \mathrm{Ft} 25 \%$ 2Fp450\&file=inebase\&L=0>. Acceso en: 7 nov. 2013.

Fundación Telefónica. La sociedad de la información en España. 2001. Disponible en: <http://www.fundacion.telefonica.com/ es/arte_cultura/publicaciones/detalle/176>. Acceso en: 7 nov. 2013.

Gobierno de Extremadura. Observatorio extremeño de la sociedad de la información. 2009. Disponible en: <http:// www.juntaex.es/consejerias/infraestructuras-desarrollotecnologico/dg-sociedad-informacion/Observatorio-idesidweb.html>. Acceso en: 7 nov. 2013.

Monge González, R.; Chacón, F. Cerrando la brecha digital en Costa Rica: acceso y uso de las tecnologías de la información y las comunicaciones en Costa Rica. San José: Fundación CAATEC, 2002. Disponible en: <http://www.caatec.org/CAATEC/ publicaciones/crdigital/CR_Digital_1.pdf>. Acceso en: 6 nov. 2013.

Monge González, R.; Hewitt, J. Tecnologías de la información y las comunicaciones (TICS) y el futuro desarrollo de Costa Rica: el desafío de la exclusión. San José: Academia de Centroamérica, 2004. Disponible en: <http://www.caatec.org/CAATEC/ publicaciones/crdigital/CR_Digital_3.pdf $>$. Acceso en: 6 nov. 2013.

Norris, P. Digital divide? Civic engagement, information poverty and the internet worldwide. Cambridge: Cambridge University Press, 2001.

Organización para la Cooperación y Desarrollo Económico. Understanding the digital divide. París: OECD Publications, 2001.

Serrano Santoyo, A.; Martínez Martínez, E. La brecha digital: mitos y realidades. México: Editorial UABC, 2003. Disponible en: <http://labrechadigital.org/labrecha/LaBrechaDigital_ MitosyRealidades.pdf>. Acceso en: 6 nov. 2013.

Unión Europea. Libro Verdesobre la convergencia de los sectores de telecomunicaciones, medios de comunicación y tecnologías de la información y sobre sus consecuencias para la reglamentación: en la perspectiva de la sociedad de la información. Bruselas: Comisión Europea, 1997.

Van Dijk, J. A framework for digital divide research. Electronic Journal of Communications, v.12, n.1/2, 2002. Available from: <http://www.cios.org/EJCPUBLIC/012/1/01211.html>. Cited: Nov. 6, 2013.

Van Dijk, J.; Hacker, K. The digital divide as a complex and dynamic phenomenon. The Information Society, v.19, n.4, p.315-326, 2003.

World Resources Institute. Creating digital dividends. 2000. Available from: <http://www.digitaldividend.org/>.Cited: Nov. 6, 2013. 
